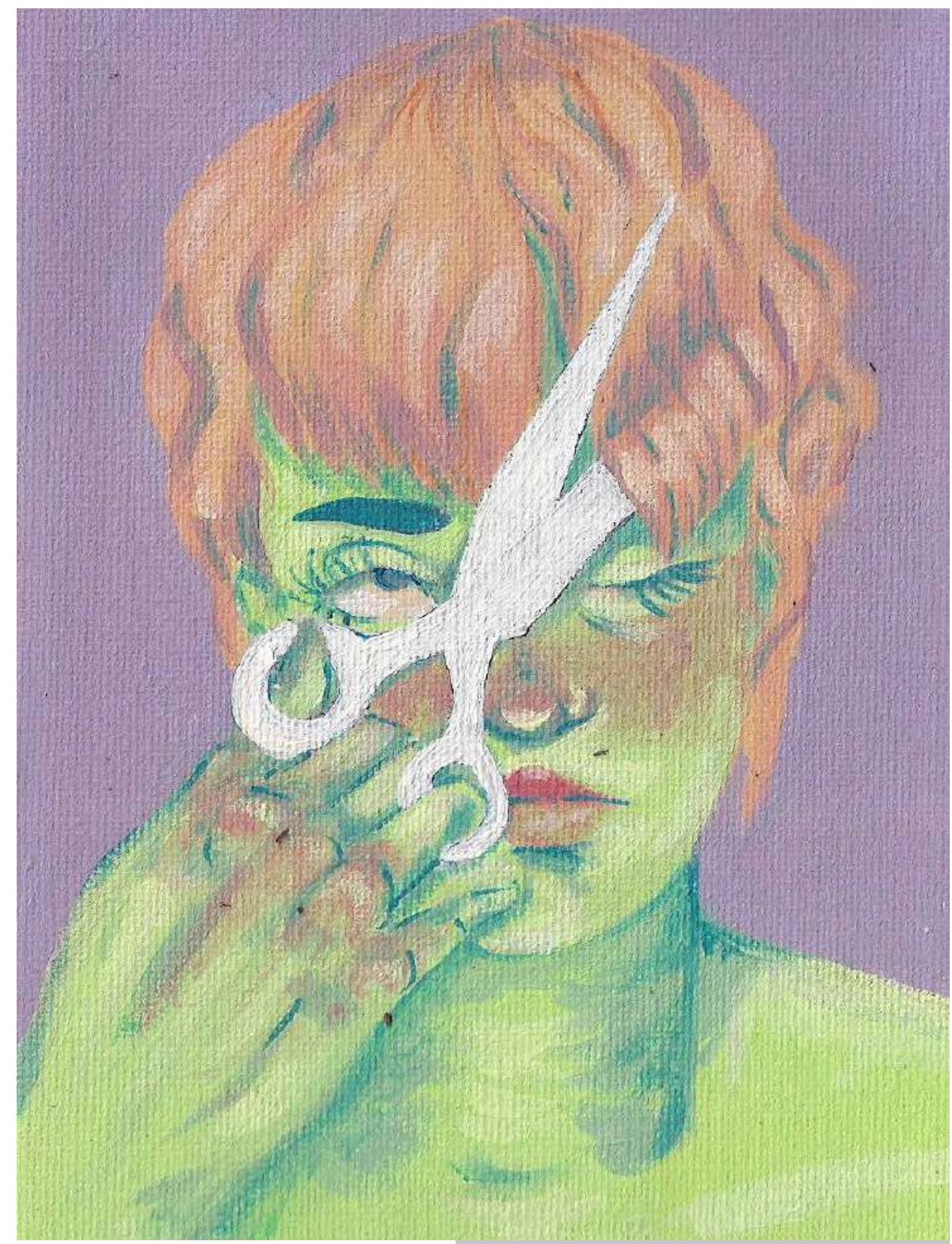

Artista invitada

Paula Alejandra García

De la serie Encierros

Acrílico sobre lienzo

2020

Cortesía Revista Ojo de Pez 


\title{
Los fundamentos ontológicos del cambio paradigmático de políticas públicas y sus implicaciones teóricas*
}

\author{
Edgar Alberto Zamora-Aviles (Ecuador) ${ }^{* *}$
}

\section{Resumen}

Este trabajo problematiza los fundamentos ontológicos de algunas explicaciones del cambio paradigmático de políticas públicas. Se realiza una revisión de literatura utilizando la tipología de fundamentos ontológicos para la explicación causal propuesta por Patrick Jackson, para mapear distintas explicaciones del cambio paradigmático de políticas públicas. Se argumenta que el desacuerdo persistente sobre el cambio paradigmático de políticas y sobre el concepto de paradigma se debe a diferencias en los fundamentos ontológicos de las teorías. Este desacuerdo es herencia parcial de Peter Hall porque en su trabajo existe ambigüedad entre dos explicaciones causales ontológicamente diferentes: una que da lugar a considerar que el cambio paradigmático puede ser incremental y otra que lo sitúa como de naturaleza diferente al aprendizaje social. En términos de teorías sustantivas, esta diferencia ontológica se expresa entre enfoques más centrados en las instituciones - y los actores - y enfoques más centrados en las ideas para explicar el cambio paradigmático de políticas públicas. Esto contribuye a aclarar ciertas discusiones sobre la interacción ideas-instituciones en los procesos de cambio gradualacumulativo teorizados recientemente.

\section{Palabras clave}

Políticas Públicas; Ontología; Neopositivismo; Realismo Científico; Neoinstitucionalismo.

Fecha de recepción: junio de 2020 - Fecha de aprobación: noviembre de 2020

\footnotetext{
* Este trabajo fue realizado en el marco de la investigación doctoral (2015-2020) como becario en la Facultad Latinoamericana de Ciencias Sociales (Flacso)-Ecuador.

** Politólogo. Magíster en Relaciones Internacionales. Doctor en Políticas Públicas. Docente de la Facultad de Ciencias Sociales y Jurídicas, Universidad Internacional SEK Ecuador (UISEK). Investigador asociado del Laboratorio Políticas Públicas Comparadas, Facultad Latinoamericana de Ciencias Sociales (Flacso)-Ecuador. Correo electrónico: alberto.zamora@uisek.edu.ec - Orcid: 00000001-6547-423X - Google Scholar: https://scholar.google.com/citations?user=cdjFsu0AAAAJ\&hl=es
} 


\title{
Cómo citar este artículo
}

Zamora-Aviles, Edgar Alberto. (2021). Los fundamentos ontológicos del cambio paradigmático de políticas públicas y sus implicaciones teóricas. Estudios Políticos (Universidad de Antioquia), 60, pp. 225-249. DOI: 10.17533/udea.espo. n60a10

\section{The Ontological Basis of Paradigmatic Public Policy Change and their Theoretical Implications}

\begin{abstract}
The work problematizes the ontological basis of some explanations about paradigmatic change in public policies. Methodologically, the article carries out a literature review using the typology of ontological foundations for the causal explanation constructed by Patrick Jackson to classify the different explanations of paradigmatic change in public policies. It is argued that disagreement persists about the paradigmatic change in policies, as well as on the concept of paradigm concept, due to the different ontological foundations at stake. It could also established that this disagreement is partially inherited from Peter Hall, since in his work we find ambiguity between two ontologically different causal explanations: one that would

[226] lead to considering that the paradigm change may be incremental, and another one that places it as different in nature from social learning. In terms of substantive theories, this ontological difference is expressed between approaches that are more focused on institutions - and actors - and approaches that are more focused on ideas to explain the paradigmatic policy change. This helps to clarify certain discussions about the interaction between ideas and institutions in the processes of gradual-cumulative change recently theorized.
\end{abstract}

\section{Keywords}

Public Policy; Ontology; Neopositivism; Scientific Realism; Neoinstitutionalism. 


\section{Introducción. El cambio de políticas públicas y la importancia de una lectura metateórica}

Cambio y desarrollo «normal» de políticas públicas son dos problemas de investigación articulados (Capano, 2009; Howlett y Cashore, 2009). En relación con los enfoques teóricos sobre políticas públicas, es factible clasificar la literatura sobre el cambio entre racionalistas, cognitivistas y neoinstitucionalistas (Fontaine 2015a; 2015b). Los enfoques racionalistas se centran en el rol de los actores y la toma de decisiones. Entre estas perspectivas pueden encontrarse el incrementalismo (Lindblom, 1959), las corrientes múltiples (Kingdon, 1995) y el equilibrio puntuado (True, Jones y Baumgartner, 2010). Entre los enfoques cognitivistas pueden incluirse las coaliciones promotoras (ACF) (Sabatier y Weible, 2010) y el enfoque de referenciales -globales y sectoriales- (Muller, 2006). Estos se centran en estudiar el papel de las ideas en los procesos de cambio de políticas.

Los enfoques neoinstitucionalistas centran su propuesta de explicación en el rol de las instituciones en el cambio de políticas y en sus relaciones con ideas y actores. Aquí se puede ubicar la «lógica de lo adecuado» (March y Olsen, 2006), la dependencia de la trayectoria (Mahoney, 2000; Pierson, 2000) y la teoría de los tres órdenes de cambio (Hall, 1993). Esta última presenta dos patrones de cambio: unos por aprendizaje social, que remiten a ajustes en y cambios de los instrumentos de política sin alterar los objetivos generales de la política — cambios de primer y segundo orden, respectivamente-; y otros cambios de naturaleza distinta, denominados cambios paradigmáticos - o de tercer orden-, que alteran los objetivos generales de las políticas y provocan cambios descendentes en los otros órdenes. Después de Peter Hall, este punto de partida teórico ha dado lugar a trabajos empíricos que construyen narrativas causales diferentes sobre el cambio paradigmático de políticas públicas.

Ya se ha llamado la atención sobre la importancia y necesaria coherencia que deben exhibir las elecciones epistemológicas y teóricas que hacen los investigadores cuando estudian el cambio de políticas (Capano, 2009; Capano y Howlett, 2009). Siguiendo la propuesta de Patrick Jackson (2011), en este artículo se sostiene que la diferenciación epistemológica no constituye un punto de partida apropiado para comprender las concepciones 
sobre la causalidad que subyacen a las explicaciones teóricas y a las metodologías usadas para explicar el cambio de políticas. Por ejemplo, nótese la confusión persistente en Giliberto Capano (2009) al distinguir entre elecciones epistemológicas y teóricas a la hora de definir la naturaleza del cambio: lineal-no lineal, adaptativo-acumulativo, evolutivo-revolucionario. Analíticamente, cualquier clasificación debe establecer límites claros entre las diferentes categorías porque allí reside su valor. Esto no desconoce que en la práctica de la producción académica los diversos trabajos sean ambiguos en sus decisiones.

Este texto se propone hacer uso del enfoque de Jackson sobre «formas de hacer ciencia» para mapear diferentes teorías-explicaciones sobre cambio paradigmático de políticas públicas que parten de una misma «raíz», el trabajo de Hall, y que, sin embargo, concluyen en procesos de cambio diferentes: cambio gradual acumulativo versus cambio paradigmático. De acuerdo con Jackson (2011), una clasificación basada en opciones ontológicas constituye una mejor guía para organizar el amplio debate sobre cambio paradigmático de políticas públicas. Así es posible distinguir a) las elecciones que los investigadores hacen sobre las formas en las que estos se enganchan al mundo - hook-up to the world-: ontología dualista o monista -mente-mundo-; y b) la concepción de la relación entre el conocimiento y la observación, la cual permite diferenciar entre fenomenalismo y transfactualismo.

La propuesta de Jackson no exhibe los problemas de determinismo que tienden a presentar otros autores en las relaciones «positivismométodos cuantitativos», «interpretativismo-métodos cualitativos» y «realismo científico-métodos mixtos» (Marsh y Furlong, 2010; Monteiro y Ruby, 2009; Yanow y Schwartz, 2006, caps.1 y 4). Parafraseando, los tipos ideales de «hacer ciencia» de Jackson no son tan irrelevantes como un suéter, pero tampoco son una piel imposible de quitar. Finalmente, la propuesta de clasificación de Jackson es completa y exhaustiva sobre los ejes analíticos, cosa que no ocurre con otras tipologías que parten de la diferenciación epistemológica antes que ontológica, distinguiendo entre «positivismo-postpositivismo» o «positivismo-interpretativismo», «positivismo-postpositivismo-enfoques críticos», entre otros (conta: Guba y Lincoln, 1994; Roth, 2008, pp. 71-75; Hall, 2003; Yanow, 2014). 


\section{1. ¿Cómo mapear los fundamentos ontológicos de las explicaciones del cambio paradigmático? La propuesta metodológica de Patrick Jackson}

En medio del debate sobre el uso de la filosofía de la ciencia para fundamentar la cientificidad de campos como las relaciones internacionales o el análisis de políticas públicas, diversos autores han señalado los límites del «argumento/postura fundacional» (Monteiro y Ruby, 2009). Jackson (2009; 2011, p. 16) suscribe este punto de partida con reservas y sobre la base del trabajo de Max Weber sostiene que el criterio de demarcación ciencia/nociencia debe ser la búsqueda de conocimiento científico en sí mismo, y la ciencia «es toda indagación empírica diseñada para producir conocimiento» (Jackson, p. 19), con carácter de sistematicidad, sometido a crítica pública y que procura hacer sentido sobre el mundo (p. 193). Jackson (2010; 2011; 2013 ; 2015) defiende una concepción pluralista de la ciencia y su recurso a la reflexión filosófica busca clarificar los términos y las implicaciones de la toma de posición frente al problema de cómo producir conocimiento científico y no evaluar dichas posiciones, las cuales son, por definición, inconmensurables.

Convencionalmente, la reflexión sobre la filosofía de la ciencia se atiene a preguntar primero por la ontología — pregunta sobre el ser: ¿qué existe en el mundo?_- luego por la epistemología — pregunta sobre el conocimiento: ¿cómo los observadores formulan y evalúan sus afirmaciones sobre el mundo? - y, por último, por la metodología —comúnmente entendida como la selección de herramientas específicas de investigación(Jackson, 2011, p. 26). Siguiendo a Sartori, la metodología es definida como «la preocupación por la estructura y los procedimientos lógicos de la investigación» (p. 25) y, por tanto, está directamente relacionada con las posiciones ontológicas. Jackson hila fino al proponer distinguir entre «ontologías filosóficas» y «ontologías científicas». Las ontologías filosóficas remiten a las «formas de conectarse con el mundo» y las ontologías científicas son «formas de ver el mundo» (p. 28). Esta distinción importa porque solo las ontologías filosóficas deben ser consideradas en el debate y, así definidas, subsumen el problema de la epistemología. ${ }^{1}$

\footnotetext{
${ }^{1}$ El problema de la epistemología solo tiene sentido si se asume una postura ontológica dualista. Este es el problema de la «ansiedad cartesiana» por cerrar la brecha entre mundo-mente (Jackson, 2011, p. 31). Para los monistas el problema de la ansiedad cartesiana desparece y con él también desaparece el «problema epistemológico».
} 
Más que posicionamientos, las metodologías se sustentan en dos «apuestas filosóficas» (Jackson, 2013; contra: Humphreys, 2013). Cualquier apuesta sobre ontología filosófica alude, por lo menos, a tres cosas: al investigador, al mundo que debe ser investigado y al carácter de la relación entre ambos. Así, los ejes de la tipología, las dos apuestas sobre ontologías filosóficas son: a) la forma en que el investigador se conecta con el mundo que cual permite diferenciar entre dualismo y monismo; $y$ b) la forma que adquiere la relación entre el conocimiento y la observación — directa- que permite diferenciar entre fenomenalismo y transfactualismo - el conocimiento se produce solamente sobre objetos que pueden ser observados directamente o también es posible producir conocimiento sobre objetos que son, en principio, no-observables - . Los cuatro tipos ideales de metodologías — comprendidos como tipos ideales de hacer ciencia- se muestran en la figura $1 .{ }^{2}$

Figura 1. Tipos ideales de metodologías en función de las apuestas filosóficas.

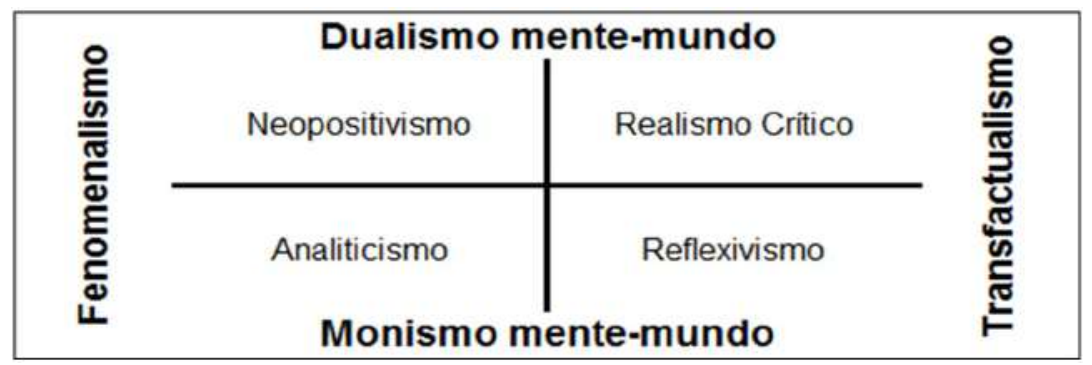

Fuente: elaboración propia a partir de Jackson (2011).

Como consecuencia de las apuestas filosóficas, estas cuatro metodologías se diferencian en varios aspectos: a) la forma en la que cada una trata de garantizar-justificar sus afirmaciones sobre el conocimiento; b) su comprensión de la causalidad y de la explicación causal; y c) el uso que hacen de la comparación entre casos (Jackson, 2011, p. 197). En cuanto a la primera de estas cuestiones, para el neopositivismo las afirmaciones de conocimiento válidas son aquellas que permanecen como conjeturas no

\footnotetext{
${ }^{2}$ Sin duda, cualquier lector notará la ausencia del «constructivismo» en esta tipología. Siguiendo su crítica contra Nuno Monteiro y Keven Ruby (2009), Jackson (2009; 2011, pp. 203-204) afirma que «el constructivismo es, ante todo, una teoría social o una ontología científica, pero no es una consideración metodológica sobre el estatus y el carácter filosófico del conocimiento» y, por tanto, «metodológicamente hablando, el constructivismo es una categoría que no tiene sentido».
} 
falseadas y el procedimiento para evaluarlas es la prueba de hipótesis. Para el realismo crítico el conocimiento válido es la mejor aproximación existente al mundo y esta se evalúa a través de investigaciones de laboratorio o de argumentos trascendentales — sobre aspectos no observables del mundo-. El analiticismo toma como válido el conocimiento que es útil —nótese la relación con el pragmatismo- y evalúa las afirmaciones sobre la base de la construcción de narrativas analíticas. El reflexivismo asume como válido el conocimiento que constituye un dispositivo para mejorar la autoconsciencia y procede a evaluar sus afirmaciones teorizando sobre las propias condiciones sociales del investigador.

El tipo de causalidad del neopositivismo es la generalización empírica y se procede a ella a través de la subsunción de situaciones específicas en leyes generales. Para el realismo crítico la causalidad es «disposicional» dispositional- y la explicación causal normalmente procede a determinar las condiciones tipo INUS en una situación específica —condiciones causales individualmente insuficientes, pero necesarias como parte de un complejo causal que es innecesario pero suficiente para producir un resultado causal(Jackson, 2011, p. 199); esta es una explicación de naturaleza «dependiente del contexto» y, por tanto, rechaza la idea de ley general. El analiticismo conceptualiza la causalidad como «ideal-típica» y configurativa, no como un asunto de generalización empírica, sino de generalidad lógica y la explicación causal se construye mediante el uso de los tipos-ideales para esclarecer aspectos específicos contingentes, haciendo uso de contrafactuales. El reflexivismo es el menos interesado en la causalidad, pero si es posible conceptualizarla adquiere la forma de relación dialéctica entre las condiciones sociales y los esfuerzos explícitos por delinearlas. Las explicaciones causales reflexivistas se concentran en poner en evidencia tensiones irresueltas en el conocimiento existente (p. 200).

Respecto al uso de la comparación en cada una de estas metodologías, en el neopositivismo esta adquiere importancia para construir leyes generales, poniendo a prueba las hipótesis de covariación entre la mayoría de los casos posibles. En el realismo científico la comparación adquiere importancia como forma de contrastar el funcionamiento específico de las configuraciones causales tipo INUS, pero no aspira a generalizaciones ni predicciones. Para el analiticismo la comparación es pragmática, se usa para mostrar la utilidad de los tipos-ideales construidos y por eso es una especie de «comparación 
individualizada». Finalmente, para el reflexivismo la comparación busca contribuir al cambio social porque busca poner en evidencia la artificialidad — «no-naturalidad»— de los supuestos sobre el orden social Jackson, 2011, pp. 200-201).

\section{La manzana de la discordia: ambigüedad originaria del cambio paradigmático en Peter Hall}

Dentro de los debates sobre el Estado, Hall (1993) procura analizar la naturaleza del «aprendizaje social» y el proceso por el cual cambian las políticas (p. 276). Las teorías coinciden en que el aprendizaje social permite explicar el cambio de políticas públicas, sin embargo, difieren en la manera en que se produce el proceso de aprendizaje social. Para los más estadocéntricos se desarrolla dentro del aparato estatal y para los más estructuralistas involucra el conjunto de actores del sistema político, y no solo el aparato estatal. Hall define el aprendizaje social como «una intención deliberada de ajustar las metas o técnicas de la política pública en respuesta a la experiencia y la nueva información. El aprendizaje se produce cuando la política pública cambia como resultado de dicho proceso $»^{3}$ (p. 278).

[232] Un aporte significativo de este trabajo es distinguir tipos de cambio de políticas (Hall, 1993, pp. 278-279). Cambios de primer orden implican ajustes en los instrumentos y cambios de segundo orden implican cambios de los instrumentos de política. Estos dos cambios se producen por procesos de aprendizaje social, esa es su naturaleza. Los cambios de tercer orden alteran los objetivos generales de las políticas: «Estos cambios ocurren raramente, pero cuando ocurren como resultado de reflexión sobre la experiencia pasada, podemos describirlos como cambios de tercer orden» (p. 279). Los cambios de primer y segundo orden no conducen necesariamente a cambios de tercer orden (p. 291), mientras que estos últimos sí ocasionan cambios descendentemente; sin embargo, el cambio de tercer orden es de naturaleza diferente y no encaja en el patrón de cambio como aprendizaje social (p. 287). En esta diferenciación es donde emerge la noción de paradigma de política.

Los hacedores de política siempre actúan desde un marco referencial de ideas que no es puesto en cuestión: un paradigma de política (p. 279).

\footnotetext{
${ }^{3}$ La traducción directa de esta y las demás citas textuales del inglés son responsabilidad exclusiva del autor de este artículo.
} 
Aquí Hall recurre a la analogía de Thomas Kuhn (2004) sobre la forma en que se desarrolla la ciencia. Cambios de primer y segundo orden pueden ser vistos como casos de «hechura de políticas normal», mientras que cambios de tercer orden «reflejan un proceso diferente, marcado por cambios radicales en los términos generales del discurso de la política, asociados con un cambio de paradigma» (Hall, 1993, pp. 279 y 291). Por definición, los paradigmas son inconmensurables y esto tiene tres implicaciones: a) el proceso de cambio de paradigma tiene un carácter más sociológico y político que científico, esto implica que la lucha por el cambio de paradigma se desarrolla en la arena política y, por tanto, se desarrolla también como una lucha de poder entre actores (Hall, 1993, pp. 280, 289 y 292); b) el cambio de paradigma esta precedido por un cambio en el locus de autoridad sobre el campo de política de que se trate; y c) en el proceso de cambio de paradigma son importantes los escenarios de experimentación y fallas de política.

Al cambio paradigmático de Hall subyace una paradoja explicativa, relacionada con el concepto de autoridad de los paradigmas, con su propiedad de inconmensurabilidad y con el rol que desempeñan las fallas del viejo paradigma (Blyth, 2013, p. 202; Hogan y Howlett, 2015b). Esta paradoja puede ser comprendida como resultado de los préstamos teóricos tomados de Kuhn (Sankey, 1993; Daigneault, 2014; 2015; Zittoun, 2009; 2015). Como consecuencia del rol asignado a la autoridad, a las fallas-errores y a la inconmensurabilidad en la explicación de Hall sobre cambio de políticas, es posible afirmar que existen dos lógicas de explicaciones causales yuxtapuestas e inconmensurables (Blyth, 2013).

En el primer caso, la explicación del cambio de tercer orden puede tomar la forma de cambio incremental acumulativo - gobernado por aprendizaje social—, soportado en las fallas recurrentes del viejo paradigma de política; en el segundo caso, el cambio de tercer orden toma la forma de cambio radical — ruptura con viejo paradigma—, gobernado por procesos «más políticos y sociales, y menos científicos» que el aprendizaje social. En la primera explicación la tesis de inconmensurabilidad es débil y los aspectos relacionados con las fallas del viejo paradigma desempeñan un rol más significativo; en la segunda explicación la tesis de inconmensurabilidad es fuerte y las fallas de política no son solo «hechos», sino que están directamente relacionadas con la lucha autoritativa sobre la interpretación de dichos hechos como fallas _locus de autoridad_. En la terminología de Jackson (2011), 
estas dos narrativas causales existen porque parten de diferentes metodologías o formas de hacer ciencia.

\section{Un mapeo de las explicaciones sobre los paradigmas de políticas y el cambio paradigmático después de Peter Hall}

El siguiente mapeo se construyó sobre las diferentes formas de investigar y explicar el cambio paradigmático de políticas públicas; sin embargo, la literatura revisada no necesariamente se concentra de manera específica en este tema y algunos trabajos solo abordan aspectos relacionados con los paradigmas de políticas — la conceptualización de los paradigmas, los procesos mediante los cuales una idea adquiere estatus de paradigma, entre otros-, pero son útiles para ejemplificar las diversas metodologías o formas de hacer ciencia. ${ }^{4}$ Siguiendo estrictamente a Hall, la mayoría de la literatura revisada es dualista en términos ontológicos. Vale aclarar que este no es un mapeo de «autores y su obra», sino de algunos trabajos específicos de cada autor, debido a que un mismo investigador puede variar sus apuestas ontológicas a lo largo de su trayectoria.

\subsection{Neopositivismo: redefiniendo los paradigmas y el cambio paradigmático de políticas para observarlos directamente}

La literatura en esta metodología busca construir una definición operacional del concepto de paradigma de política que permita su observación directa. Este proceso tiene aparejadas dos ideas clave: a) las políticas públicas —objeto de estudio- existen independientemente del investigador —dualismo—; y b) toda explicación científica debe estar basada en la observación directa de la evidencia empírica — fenomenalismo—. En este segundo aspecto importa especificar el tipo de observaciones esperadas para corroborar la existencia tanto de los paradigmas como de los cambios paradigmáticos. Este enfoque es el más frecuente.

\footnotetext{
${ }^{4}$ En general, la literatura revisada se concentra en cuatro obras colectivas: el número especial sobre cambio de políticas públicas de la Journal of Comparative Policy Analysis (Vol. 11, N. ${ }^{\circ}$ 1, 2009); el número especial conmemorativo de las dos décadas del trabajo de Hall de la revista Governance (Vol. 26, N.․ 2, 2013); el simposio sobre paradigmas de políticas reproducido en la Journal of European Public Policy (Vol. 21, N. ${ }^{\circ} .3$, 2014); y el conjunto de trabajos sobre los paradigmas de política incluidos en el libro editado por John Hogan y Michael Howlett (2015a).
} 
Michael Howlett y Benjamin Cashore (2009) señalan que el trabajo de Hall (1993) rompe el dominio de la «vieja ortodoxia» del incrementalismo como patrón de cambio de políticas; sin embargo, desarrollos posteriores sobre Hall consolidaron una «nueva ortodoxia», representada por el modelo de «equilibrio puntuado». En este modelo «homeostático», solo un shock externo explica el cambio de tercer orden — paradigmático- a través de la des-institucionalización de un equilibrio previo (Howlett y Cashore, 2009, p. 36). Pero esta nueva ortodoxia evidencia problemas sobre dos asuntos: primero, están los elementos de las políticas que pueden ser susceptibles de cambio - problema de la variable dependiente-; segundo, sería necesario establecer nuevas tipologías de cambio. En el primer aspecto se ha propuesto caracterizar las políticas de acuerdo a tres niveles de abstracción, ${ }^{5}$ pero distinguiendo dos aspectos: fines y medios (Cashore y Howlett, 2007, p. 536). El resultado es una matriz de caracterización de políticas de seis dimensiones que permitiría mejores respuestas a la pregunta ¿qué cambia cuando se afirma que cambian las políticas públicas?

En el segundo aspecto se sostiene que la clásica tipología que combina la velocidad — tempo: rápido, lento— con el modo del cambio —incremental, paradigmático — falla en dos asuntos: por un lado, el modo de cambio viene a ser simultáneamente variable dependiente e independiente; y por otro lado, al fijarse en el tempo se ignora la direccionalidad del cambio — que puede ser acumulativa o adaptativa-. Así, se propone una taxonomía alternativa de los patrones de cambio de políticas: homeostático, neohomeostático, cuasihomeostático y termostático (Howlett y Cashore, 2009, pp. 41-42), que se relacionan con las tendencias de cambios difusos-graduales pero acumulativos que han trabajado otros neoinstitucionalistas — drift, conversion, layering, displacement- (Mahoney y Thelen, 2010).

Sobre la base de esta matriz que especifica la variable dependiente, Matt Wilder (2015) contribuye a operacionalizar las relaciones causales. En términos de teoría sustantiva — no de metateoría—, Wilder señala que en el análisis de políticas solo puede mantenerse el concepto de paradigma en su forma blanda, lo cual implica rechazo de la tesis de inconmensurabilidad (pp. 23-25). Esto,

\footnotetext{
${ }^{5}$ En el marco de la misma metodología neopositivista se ha propuesto una caracterización de cuatro niveles de los paradigmas de política pública: a) ideas sobre el campo de política y sobre cómo debe ser gobernado; b) objetivos de política; c) instrumentos de política; y d) las instituciones de gobernanza. El cambio paradigmático para estos autores consiste en el cambio significativo en todos los niveles (Kern, Kuzemko y Mitchell, 2015, p. 271).
} 
además, sería necesario para concebir cambios incrementales acumulativos — del primer al tercer orden-. Así, rechazar la tesis de inconmesurabilidad - en términos de teoría sustantiva - es la condición necesaria para poder evidenciar-observar directamente los cambios paradigmáticos —en términos metateóricos-.

La operacionalización del problema de los paradigmas se sintetiza en la formulación $Y \rightarrow(A * B){ }^{*} C * D^{*} E$ (Wilder, 2015, pp. 28-30). En la parte inferior de la figura 2, en los instrumentos, existe una determinación desde el nivel de abstracción mayor hacia el menor. Y en cuanto a las relaciones causales, «El "paradigma" es operacionalizado como la secuencia de notaciones "*", o la creencia en que la calibración de instrumentos $A$ contribuirá a que los instrumentos de política $B$ alcancen los objetivos instrumentals $C$ que llevarán al cumplimiento de los objetivos programáticos $D$ que, a su vez, satisfarán los objetivos paradigmáticos $E \gg$ (p. 29).

Figura 2. Matriz de desagregación de las políticas —variable dependientey operacionalización del cambio paradigmático.

\section{Policy Ends}

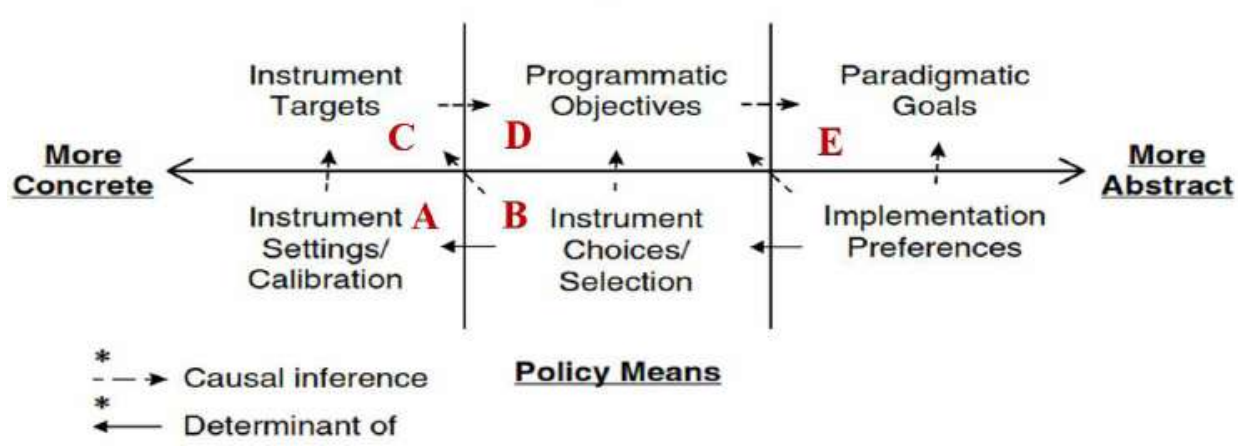

Fuente: elaboración propia a partir de Wilder (2015, p. 29).

Una nueva conceptualización de los paradigmas es la ruta seguida por Pierre-Marc Daigneault $(2014 ;$ 2015) en el debate. Su balance de la discusión señala que los paradigmas están subteorizados, que el análisis causal es débil, que existen problemas con la ausencia o debilidad de la agencia y que persiste un problema transversal de metodología que consiste en equiparar el cambio de paradigma con el cambio de políticas, hecho que lleva implícitamente 
a un «enfoque de ideas reveladas» (2015, pp. 47-49). Los paradigmas de política pública se definen como «un conjunto coherente de ideas cognitivas y normativas, sostenidas intersubjetivamente por las personas en una comunidad de políticas públicas, sobre la naturaleza de la realidad, la justicia social y el rol del estado, el problema que requiere intervención pública, los fines y objetivos que la política debe buscar, y los "instrumentos" de política para alcanzar tales fines» (2014, pp. 461-462).

Este concepto sirve al autor para aclarar fundamentalmente los problemas entre ontología y metodología a la hora de estudiar los paradigmas de política: primero, queda claro que la ontología de los paradigmas es «ideacional»: ${ }^{6}$ «los paradigmas están formados solo por ideas, mientras que las políticas sí tienen una dimensión material» (Daigneault, 2015, p. 50); segundo, los paradigmas están formados por cuatro tipos de ideas, correspondientes con cuatro dimensiones constitutivas del paradigma, la dimensión institucional no es constitutiva de los paradigmas; tercero, un paradigma debe evidenciar cierto grado mínimo de coherencia lógica y compatibilidad entre el conjunto de ideas; y quinto, las ideas que integran un paradigma deben ser ampliamente compartidas (p. 51).

El cambio paradigmático se define como un «cambio significativo» en las cuatro dimensiones constitutivas del paradigma (Daigneault, 2014). A pesar del esfuerzo, el autor reconoce que persisten desafíos de medición con esta definición (2015, pp. 55-56): ¿cómo establecer un umbral para definir el «nivel mínimo de coherencia lógica entre las ideas» ${ }^{7}$ o para establecer el grado en que las ideas son «ampliamente compartidas» por los actores?, ¿cómo evitar los problemas para acceder a las creencias o ideas que los actores sostienen?, ¿es útil construir tipos ideales de paradigmas para después evaluarlos empíricamente?

Por último, otros trabajos representativos del neopositivismo se han concentrado en la disputa política sobre las «anomalías-fallas» de las políticas (Wilder y Howlett, 2015; Baumgartner, 2014; 2013). Contrario a Hall, para

\footnotetext{
${ }^{6}$ Traducción casi literal del vocablo ideational, para no inducir a confusiones con el término «idealidealista» del castellano.

${ }^{7}$ El trabajo de Florian Kern, Caroline Kuzemko y Catherine Mitchell (2015, p. 284) incluso ha criticado la «coherencia interna de un paradigma» como dimensión constitutiva del concepto y defiende una naturaleza mixta de los paradigmas. Véase también Mitchell Orenstein (2013).
} 
Frank Baumgartner (2013) los cambios de primer-segundo-tercer orden solo evidencian diferencias de grado y no de naturaleza. Todos son expresión de un mismo proceso-patrón: la erosión de autoridad o erosión del statu quo. Como es propio del equilibrio puntuado, esta naturaleza única del cambio es probada por Baumgartner (2013, pp. 243-247) analizando la frecuencia del tipo de cambio-variación en la asignación presupuestaria en series de tiempo. Para probar que existe el mismo patrón de cambio en los tres órdenes de Hall, Baumgartner (des)agrega los datos presupuestarios según gobierno federal tercer orden-, «funciones» definidas por Office of Management and Budget $(\mathrm{OMB})$ — segundo orden-y las «subfunciones» — primer orden-. Más que una teoría de los paradigmas de política, se requiere una teoría de las ideas en las políticas públicas (Baumgartner, 2014, p. 478) que trate las ideas como variables susceptibles de ser medidas separadamente de los actores, las instituciones y las políticas, y que permita medir el poder subyacente a cada idea.

Matt Wilder y Michael Howlett (2015, p. 102) proponen un análisis político de las anomalías de las políticas públicas de carácter hermenéutico. La condición necesaria para poder desarrollar este análisis es concebir el cambio paradigmático como gradual, evolutivo antes que revolucionario y, por tanto, rechazar la tesis de inconmensurabilidad (p. 110). Las anomalías «son discrepancias entre los resultados evidenciados y los resultados esperados de la experimentación científica o de políticas públicas, que ponen en duda la veracidad del diagnóstico del problema y la eficacia de las soluciones propuestas. Las anomalías constituyen observaciones de la realidad que se salen de un marco paradigmático específico, y por tanto requieren una modificación del paradigma con el objetivo de adaptarlo» (p. 104).

El asunto clave con las anomalías es que podrían ser incorporadas al paradigma dominante — bricolage- Ante acumulación de anomalías en el paradigma dominante, la coalición que lo soporta promoverá cambios de primer-segundo orden para enfrentar dichos desafíos. Estos cambios constituyen «estrategias contingentes» — proceso de experimentación- ya que las anomalías se encuentran fuera del paradigma dominante. Si estas nuevas decisiones de política resuelven las anomalías y son susceptibles de ser incorporados, entonces se produce una ampliación del paradigma en sí mismo a través de este proceso de mixtura (Kern, Kuzemko y Mitchell, 2015; Orenstein, 2013). Si los nuevos elementos no resuelven las anomalías se produce erosión de la autoridad del viejo paradigma, acompañada de la 
búsqueda de nuevas ideas por fuera del Gobierno, hecho que lleva a una batalla por la institucionalización del nuevo paradigma (Wilder y Howlett, 2015, p. 107).

\subsection{Realismo crítico: explicar el ascenso y caída de las ideas y los paradigmas}

Fue Mark Blyth (2013) quien marcó claramente la paradoja subyacente a Hall (1993) entre dos lógicas de explicación causal: a) una de corte neopositivista, que concibe un proceso de cambio incremental acumulativo hasta el tercer orden y, por tanto, que rechaza la tesis de inconmensurabilidad; y b) otra que puede ser ubicada en el realismo científico, que se sustenta en la diferencia de naturaleza del cambio paradigmático — siendo autónomo de cambios de primer-segundo orden-y que lo explica en función de procesos políticos y sociales de mayor alcance, alrededor de la disputa autoritativa sobre la interpretación de las «fallas de política» como fallas. Blyth (2013) se ubica en esta segunda forma de explicación causal al analizar por qué la crisis de 2008-2009 no ha producido un cambio de paradigma.

La lucha sobre los paradigmas puede ser independiente de los cambios de primer y segundo orden debido a que estos cambios deben ser mediados no solo a través del paradigma dominante en un momento dado, sino a través de diversos escenarios institucionales cuyos reclamos de autoridad se sustentan en la defensa del paradigma. La lucha sobre el tercer orden es la lucha sobre el significado de las anomalías, no sobre su existencia. La inconmensurabilidad garantiza que el significado sea siempre disputable, mientras que las consecuencias distributivas del cambio de paradigma garantizan la existencia de muchos incentivos para respaldar y oponerse a tales cambios. La autoridad, en tales casos, es quizás lo más importante (p. 211. Énfasis añadido).

Nótese que, en términos de teoría sustantiva, el rechazo a la tesis de inconmensurabilidad y la posibilidad del cambio incremental acumulativo es una evidencia de la opción metodológica neopositivista según Jackson. Mantener la tesis de inconmensurabilidad implica que la explicación del cambio paradigmático permanece en cierta medida como no-observable directamente. Esta modalidad de cambio paradigmático, de rupturarevolucionario, se sustenta, parcialmente, en un argumento trascendente transfactual-: la tesis de inconmensurabilidad fuerte que haría que el proceso explícito de transición no fuera directamente observable, sino que solamente 
se pueda observar o reconstruir a posteriori, observando directamente los efectos que produce, observando cambios de primer-segundo orden.

En cierta medida, el trabajo de Blyth (2013) es similar al de Wilder y Howlett (2015). Estos trabajos representan desafíos para establecer un umbral de diferenciación entre neopositivismo y realismo según Jackson. El énfasis de Wilder y Howlett en el carácter hermenéutico del proceso que desarrollan los actores sobre las anomalías es comparable con el énfasis de Blyth en el carácter autónomo del proceso de cambio paradigmático gobernado por procesos políticos sobre la interpretación de las fallas de política o anomalías. El trabajo de Wilder y Howlett (2015) se ubica en el neopositivismo por la relación con otros trabajos aquí también revisados (Howlett y Cashore, 2009; Cashore y Howlett, 2007; Wilder, 2015) que claramente evidencian tanto el dualismo como el fenomenalismo, este último mediante un instrumental capaz de hacer observables directamente los conceptos y las relaciones causales.

Por otro lado, aunque se ha optado por ubicar este trabajo de Blyth en el realismo científico, su afirmación de que las anomalías o fallas de políticas importan por su interpretación y no por su existencia, desafía la decisión.

[240] Una forma de solventar este desafío es considerar que el estatus ontológico de las anomalías es un debate que Blyth propone en el campo de la ontología científica, no de la ontología filosófica. Esto es factible si se atiene a que el problema de Blyth con las anomalías reside en la teoría sustantiva, no en la metateoría.

El trabajo de Robert Cox y Daniel Béland (2013) también se considera claramente representativo de la metodología realista (Jackson, 2011, cap. 4). Sumándose al desafío de teorizar el poder diferenciado de las ideas (Baumgartner, 2013; 2014; Berman, 2013; Béland y Cox, 2013), preguntan: ipor qué y cómo una idea adquiere estatus de paradigma?; y en relación con esto, ¿por qué una idea puede ser más o menos influyente en el proceso de las políticas públicas? Para avanzar en las respuestas proponen el concepto de «valencia» (Cox y Béland, 2013, p. 308), definida como la «atractividad» - atractiveness - de una idea, es una cualidad emocional de una idea. La valencia varía en grado —alta-baja-, en tipo — positiva-negativa: genera atracción-aversión- y también puede cambiar en el tiempo. «Nuestro argumento posiciona la valencia como una cualidad de una idea de política pública y explica cómo interactúan la agencia y el contexto para hacer que 
ciertas ideas sean más atractivas y, por tanto, más influyentes que otras» ( $p$. 309).

La valencia puede contribuir a explicar la influencia «prerracional»que ejercen las ideas sobre los tomadores de decisión, podría contribuir a explicar el efecto de las ideas sobre la formación de preferencias. En esta teoría sustantiva sobre la valencia de las ideas, Cox y Béland (2013) son claramente dualistas: postulan la valencia como una cualidad «real» de las ideas, al tiempo que insisten en que las ideas no son cosas materiales (p. 313). Por otro lado, la referencia constante a la manera de investigar la valencia en la psicología social, así como en teoría microeconómica de comportamiento del consumidor, y, en específico, en el marketing-publicidad frente a los consumidores, permite señalar el carácter transfactual de la explicación causal.

Los autores señalan que se requieren cuasiexperimentos en la modalidad de grupos focales o en la modalidad de encuestas con diferentes grupos poblacionales para inferir la propiedad de valencia de las ideas de política pública (Cox y Béland, 2013, p. 324). Nótese que en este abanico de recomendaciones para trabajo empírico la valencia permanece siempre como algo no-observable directamente: solo a través del control que el investigador puede ejercer sobre las condiciones en el marco de las cuales se desarrolla un (cuasi)experimento social se puede inferir que la causa del efecto observado es la entidad hipotetizada. En esta discusión, la valencia permanece siempre en el estrato de la realidad no observable directamente, aunque pueden observarse directamente sus efectos; es como si la valencia solo fuera observable «en acción».

\subsection{Analiticismo: cuando los paradigmas de políticas no existen más allá de sus portavoces}

Algunos trabajos de Philippe Zittoun (2009; 2015) sobre cambio de políticas y su crítica al concepto de cambio paradigmático constituyen una buena representación del «analiticismo» (Jackson, 2011, cap. 5). En la parte baja de la figura 1 el elemento definitorio es el monismo y aquí va acompañado de una apuesta ontológica por el fenomenalismo: búsqueda de información directamente observable para explicaciones causales. El monismo de Zittoun (2009, p. 66) es claro en su crítica al problema metodológico de la objetivación de las políticas, porque tratan de reducirlas a alguna categoría de «hechos»: instrumentos, leyes, ideas, instituciones que puedan ser observados 
independientemente de los actores. «En otras palabras, es imposible realizar una disociación entre los hechos y las interpretaciones debido a las condiciones bajo las cuales esos "hechos" son construidos» (p. 70).

Criticando directamente a Hall, Zittoun (2015, pp. 123-127) señala que la traducción de los conceptos y la teoría de cambio revolucionario de Kuhn desde las ciencias naturales hacia las políticas públicas posee problemas debido a diferencias ontológicas entre ambos dominios: a) el dominio exclusivo de un solo grupo que se identifica con un paradigma científico no es comparable con el dominio que podría tener un solo grupo dentro de un campo de políticas; b) la conceptualización de políticas públicas como compuestas de tres elementos —valores, principios e instrumentos- no es equiparable con la estructuración de un paradigma científico como un conjunto ordenado y coherente de conocimientos, principios, leyes e ideas; c) los enigmas que desafían los paradigmas científicos no son equiparables a los problemas de política pública que desafiarían un paradigma de política; d) el vínculo entre un paradigma científico y su comunidad es claro, mientras que las posiciones de los actores que respaldan un paradigma de política pública no son fijas. Por esto Zittoun señala que la «importación» hecha por Hall de la teoría del cambio paradigmático no funciona.

Ahora, si el punto clave de los paradigmas es enfatizar el rol de las ideas en el cambio de políticas, para superar los problemas asociados a los paradigmas Zittoun (2015) propone el concepto «declaración de política».

Una declaración de política es un ensamble discursivo de componentes diferentes y heterogéneos, sin ninguna coherencia predefinida, que otorga un significado específico a una propuesta de política pública y que está soportada por algunos policymakers que desean cambiar la política [...]. Los diferentes componentes de una declaración de política pueden incluir problemas, soluciones, instrumentos, valores, ideologías, objetivos, relaciones de causalidad, consecuencias, productos, resultados, el público, responsabilidades, o culpables (p. 129).

Para Zittoun (2009; 2015) analizar el cambio de las políticas públicas significa analizar los discursos que los actores construyen sobre el cambio de políticas, la forma en que construyen coaliciones y la forma en que construyen argumentaciones sobre las fallas-éxitos de las políticas existentes. Analizar el cambio de políticas es analizar la trayectoria de estas declaraciones. El autor 
señala reiteradamente que este tipo de análisis debe realizarse siguiendo las pistas empíricas de la trayectoria de las declaraciones de políticas, siguiendo esos rastros que los actores van dejando a medida que disputan el significado de las políticas. En ningún momento Zittoun hace reclamaciones sobre la necesidad u obligación del analista de políticas de tomar posición — de manera reflexiva - frente a los «hechos» que analiza. Esto último es reiterado en algunas de las referencias que hace a la obra de Luc Boltanski y la «sociología de la crítica» como opuesta a la «sociología crítica».

\subsection{Reflexivismo: del análisis de políticas a la facilitación del aprendizaje transformativo}

Casi no se encuentran trabajos desde una metodología reflexivista sobre el problema del cambio paradigmático de políticas públicas. En el campo disciplinar del análisis de políticas la diversidad de posturasenfoques que se autodenominan «discursivos» o «constructivistas» resaltan la importancia de que Jackson haya dejado por fuera de su tipología a esta categoría. El mismo Zittoun (2009) rotula su análisis como discursivo, sin embargo, no tiene mucho en común con un trabajo como el de Frank Fischer y Alan Mandell (2012) que se denomina constructivista-interpretativista. Esta auto enunciación diversa se evidencia en el conjunto de trabajos que se incluyen en los volúmenes «típicamente» reflexivistas, bajo el rótulo del «giro argumentativo» (Fischer y Forester, 2002; Fischer y Gottweis, 2012). En el grupo de publicaciones colectivas que se ha tomado como base de este mapeo no se encontró ningún trabajo representativo de la metodología reflexivista, tampoco algún trabajo específico que discuta sobre el cambio de políticas directamente con-contra Hall.

De manera ilustrativa se presenta una perspectiva que discute parcialmente con Hall a través del concepto de aprendizaje social (Fischer y Mandell, 2012). Estos autores señalan que el aprendizaje social se ha hecho más relevante en el mundo contemporáneo por mayor incertidumbre, complejidad e inestabilidad. Estas características permiten ver al Gobierno y a sus instituciones como un «sistema de aprendizaje». Adhieren a una comprensión amplia de los paradigmas de política como creencias profundas de los actores - Gestalt- (p. 345) y conciben las políticas públicas concebidas como «acuerdos sociopolíticos creados discursivamente» (p. 348). La otra parte del argumento de estos autores la constituye el desarrollo del concepto de aprendizaje transformativo (pp. 350 y ss.), y se lo concibe como 
un aprendizaje contextualizado que, además, tiene como fin transformar los roles y las identidades de los sujetos involucrados en el proceso, a través de un proceso reflexivo, de análisis sobre la propia identidad, que involucra un compromiso con la democracia deliberativa (p. 357) en sucesivas fases de diálogo. Así, el analista o planificador se convierte en facilitador del proceso de aprendizaje transformativo que desarrollan los actores alrededor de la definición de un problema público (pp. 359-360).

\section{Consideraciones finales}

En términos de resultados generales del mapeo, el desarrollo de las investigaciones sobre paradigmas y cambio paradigmático de políticas públicas se concentra en la metodología neopositivista, por lo menos en las obras tomadas como fuentes de análisis. Pocas abordan esta misma problemática desde la metodología del realismo científico. Vale señalar que, sin duda, el trabajo de Hall (1993) es dualista —-mente-mundo—, según la clasificación de Jackson (2011), pero sobre la relación observación-conocimiento la paradoja originaria de la explicación causal podría ubicarlo tanto en el fenomenalismo como en el tranfactualismo. Por otra parte, aunque no es posible ubicar a Hall en el monismo, en el trabajo de Zittoun (2015) se observó una discusión

[244] muy directa con los paradigmas de políticas que permitió evidenciar una metodología muy distinta a la de Hall y que incluso lleva a Zittoun a proponer un nuevo concepto para reemplazar a los paradigmas. Finalmente, el texto de Fischer y Mandell (2012) es ilustrativo de la metodología reflexivista, pero no desarrolla una discusión directa con el trabajo de Hall.

Evidentemente, la propiedad de inconmensurabilidad de los paradigmas ha sido el parteaguas entre neopositivistas y realistas a la hora de analizar el cambio paradigmático de políticas públicas. Este es un elemento de teoría sustantiva que se conecta directamente con aspectos metateóricos. Citando a Hall (2016), no es necesario pensar siempre el cambio -institucional, específicamente- con la dimensión del Big Bang. Aunque la ruptura o discontinuidad entre paradigmas sea —o parezca - mínima, siempre existen elementos de ruptura que reflejan inconmensurabilidad.

En este sentido, siguiendo a Guillaume Fontaine (2015c; Fontaine, Narváez y Velasco 2017), se puede proponer una organización de este debate reconociendo que: a) los paradigmas tienen ontología — científicaideacional y también el «cambio de paradigma»; b) el «cambio paradigmático 
de políticas públicas» es un proceso complejo que incorpora el cambio de paradigma y se concreta como cambio en elementos ideacionales, pero también materiales-institucionales de las políticas públicas; y c) el cambio de tercer orden de políticas públicas es la forma directamente observable del cambio paradigmático de políticas, tiene ontología — científica- institucional como cambio global en el conjunto de instrumentos de las políticas públicas. Esta sería una perspectiva más realista que neopositivista, en la que el proceso general a explicar es el «cambio paradigmático de políticas públicas», que en sí mismo permanece como no-observable directamente, al menos parcialmente.

Este mapeo, susceptible de ser mejorado ampliamente, es de todas maneras ilustrativo de las diferentes formas que puede tomar la indagación científica. Como tal, aporta sobre la pertinencia de la tipología construida por Jackson. También refuerza la utilidad de la distinción entre ontologías científicas y filosóficas, hecho que se vio evidenciado en las discusiones sobre las implicaciones que las apuestas filosóficas tenían para las teorías sustantivas del cambio de políticas. Por supuesto que la utilidad o validez de este tipo de trabajos solo puede ser reconocida sobre la base de una concepción pluralista de la ciencia, en la que se demanda mayor coherencia entre las apuestas ontológicas y las teorías sustantivas del cambio de políticas.

\section{Referencias bibliográficas}

1. Baumgartner, Frank. (2013). Ideas and Policy Change. Governance, 26 (2), pp. 239-258. https://doi.org/10.1111/gove.12007

2. Baumgartner, Frank. (2014). Ideas, Paradigms and Confusions. Journal of European Public Policy, 21 (3), pp. 475-480. https://doi.org/10.1080/13501763.201 3.876180

3. Béland, Daniel \& Cox, Robert. (2013). The Politics of Policy Paradigms. Governance, 26 (2), pp. 193-195. https://doi.org/10.1111/gove.12034

4. Berman, Sheri. (2013). Ideational Theorizing in the Social Sciences since «Policy Paradigms, Social Learning, and the State». Governance, 26 (2), pp. 217-237. https://doi.org/10.1111/gove.12008

5. Blyth, Mark. (2013). Paradigms and Paradox: The Politics of Economic Ideas in Two Moments of Crisis. Governance, 26 (2), pp. 197-215. https://doi.org/10.1111/ gove. 12010

6. Capano, Giliberto. (2009). Understanding Policy Change as an Epistemological and Theoretical Problem. Journal of Comparative Policy Analysis: Research and Practice, 11 (1), pp. 7-31. https://doi.org/10.1080/13876980802648284 
7. Capano, Giliberto \& Howlett, Michael. (2009). Introduction: The Determinants of Policy Change: Advancing the Debate. Journal of Comparative Policy Analysis: Research and Practice, 11 (1), pp. 1-5. https://doi.org/10.1080/13876980802648227

8. Cashore, Benjamin \& Howlett, Michael. (2007). Punctuating Which Equilibrium? Understanding Thermostatic Policy Dynamics in Pacific Northwest Forestry. American Journal of Political Science, 51 (3), pp. 532-551. https://doi. org/10.1111/j.1540-5907.2007.00266.x

9. Cox, Robert \& Béland, Daniel. (2013). Valence, Policy Ideas, and the Rise of Sustainability. Governance, 26 (2), pp. 307-328. https://doi.org/10.1111/gove.12003

10. Daigneault, Pierre-Marc. (2014). Reassessing the Concept of Policy Paradigm: Aligning Ontology and Methodology in Policy Studies. Journal of European Public Policy, 21 (3), pp. 453-469. https://doi.org/10.1080/13501763.2013.834071

11. Daigneault, Pierre-Marc. (2015). Can You Recognize a Paradigm When You See One? Defining and Mesuring Paradigm Shift. In: Hogan, John \& Howlett, Michael (Eds.). Policy Paradigms in Theory and Practice. Discourses, Ideas and Anomalies in Public Policy Dynamics (pp. 43-60). New York: Palgrave Macmillan. https://doi. org/10.1057/9781137434043_3

12. Fischer, Frank \& Forester, John. (2002). The Argumentative Turn in Policy Analysis and Planing. London: UCL. https://doi.org/10.4324/9780203499467

13. Fischer, Frank \& Gottweis, Herbert (Eds.). (2012). The Argumentative Turn Revisited. Public Policy as Communicative Practice. Durham \& London: Duke [246] University. https://doi.org/10.1515/9780822395362

14. Fischer, Frank \& Mandell, Alan. (2012). Transformative Learning in Planning and Policy Deliberation. Probing Social Meanings and Tacit Assumptions. In: Fischer, Frank \& Gottweis, Herbert (Eds.). The Argumentative Turn Revisited. Public Policy as Communicative Practice (pp. 343-370). Durham \& London: Duke University. https:// doi.org/10.1215/9780822395362-012

15. Fontaine, Guillaume. (2015a). Lecciones de América Latina sobre las dimensiones racionales, cognitivas e institucionales del cambio de políticas. Presentación del dossier. ĺconos, 53, pp. 11-30. https://doi.org/10.17141/ iconos.53.2015.1518

16. Fontaine, Guillaume. (2015b). Análisis de políticas públicas. Conceptos, teorías y métodos. Madrid-Quito: Anthropos, Flacso-Ecuador.

17. Fontaine, Guillaume. (2015c). Los aportes del neoinstitucionalismo al análisis de políticas públicas. Revista Andina de Estudios Políticos, v (2), pp. 80-98.

18. Fontaine, Guillaume; Narváez, Iván \& Velasco, Susan. (2017). Explaining a Policy Paradigm Shift: a Comparison of Resource Nationalism in Bolivia and Peru. Journal of Comparative Policy Analysis: Research and Practice, 20 (2), pp. 142-157. https://doi.org/10.1080/13876988.2016.1272234 
19. Guba, Egon \& Lincoln, Yvanna. (1994). Competing Paradigms in Qualitative Research. In: Denzin, Norman \& Lincoln, Yvanna (Eds.). Handbook of Qualitative Research (pp. 105-117). Thousand Oaks: SAGE.

20. Hall, Peter. (1993). Policy Paradigms, Social Learning and the State: The Case of Economic Policymaking in Britain. Comparative Politics, 25 (3), pp. 275-296. https://doi.org/10.2307/422246

21. Hall, Peter. (2003). Aligning Ontology and Methodology in Comparative Research. In: Mahoney, James \& Rueschemeyer, Dietrich (Eds.). Comparative Historical Analysis in the Social Sciences (pp. 373-404). Cambridge: Cambridge University. https://doi.org/10.1017/CBO9780511803963.012

22. Hall, Peter. (2016). Politics as a Process Structured in Space and Time. In: Fioretos, Orfeo; Falleti, Tulia \& Sheingate, Adam (Eds.). The Oxford Handbook of Historical Institutionalism (pp. 31-50). Oxford: Oxford University. [Digital Edition]. https://doi.org/10.1093/oxfordhb/9780199662814.013.2

23. Hogan, John \& Howlett, Michael. (2015a). Policy Paradigms in Theory and Practice. Discourses, Ideas and Anomalies in Public Policy Dynamics. New York: Palgrave Macmillan.

24. Hogan, John \& Howlett, Michael (Eds.). (2015b). Reflections on Our Understanding of Policy Paradigms and Policy Change. In: Policy Paradigms in Theory and Practice. Discourses, Ideas and Anomalies in Public Policy Dynamics (pp. 3-18). New York: Palgrave Macmillan. https://doi.org/10.1057/9781137434043_1

25. Howlett, Michael \& Cashore, Benjamin. (2009). The Dependent Variable Problem in the Study of Policy Change: Understanding Policy Change as a Methodological Problem. Journal of Comparative Policy Analysis: Research and Practice, 11 (1), pp. 33-46. https://doi.org/10.1080/13876980802648144

26. Humphreys, adam. (2013). Applying Jackson's Methodological Ideal-Types: Problems of Differentiation and Classification. Millenium, 41 (2), pp. 290-308. https:// doi.org/10.1177/0305829812463476

27. Jackson, Patrick. (2009). A Faulty Solution to a False(ly Characterized) Problem: a Comment on Monteiro and Ruby. International Theory, 1 (3), pp. 455-465. https:// doi.org/10.1017/S1752971909990145

28. Jackson, Patrick. (2010). Pluralizing Social Science. Qualitative \& MultiMethod Research, 8 (1), pp. 18-23.

29. Jackson, Patrick. (2011). The Conduct of Inquiry in International Relations. Philosophy of Science and its Implications for the Study of World Politics. New York: Routledge. https://doi.org/10.4324/9780203843321

30. Jackson, Patrick. (2013). Preparing the Ground for a More Hospitable International Relations. Millenium, 41 (2), pp. 367-378. https://doi. org/10.1177/0305829812466712 
31. Jackson, Patrick. (2015). Must International Studies Be a Science? Millenium, 43 (3), pp. 942-965. https://doi.org/10.1177/0305829815579307

32. Kern, Florian; Kuzemko, Caroline \& Mitchell, Catherine. (2015). How and Why Do Policy Paradigm Change; and Doies It Matter? The Case of UK Energy Policy. In: Hogan, John \& Howlett, Michael (Eds.). Policy Paradigms in Theory and Practice. Discourses, Ideas and Anomalies in Public Policy Dynamics (pp. 269-292). New York: Palgrave Macmillan. https://doi.org/10.1057/9781137434043_13

33. Kingdon, John. (1995). Agendas, Alternatives, and Public Policies. New York: Longman.

34. Kuhn, Thomas. (2004). La estructura de las revoluciones científicas. Buenos Aires: Fondo de Cultura Económica.

35. Lindblom, Charles. (1959). The Science of «Muddling Through». Public Administration Review, 19 (2), pp. 79-88. https://doi.org/10.2307/973677

36. Mahoney, James. (2000). Path Dependence in Historical Sociology. Theory and Society, 29, pp. 507-548. https://doi.org/10.1023/A:1007113830879

37. Mahoney James \& Thelen, Kathleen. (2010). Explainig Institutional Change. Ambiguity, Agency, and Power. Cambridge: Cambridge Univertity. https://doi. org/10.1017/CBO9780511806414

38. March, James \& Olsen, Johan. (2006). The Logic of Appropriateness. In: Moran, Michael; Rein, Martin \& Goodin, Robert (Eds.). The Oxford Handbook of Public Policy (pp. 689-708). Oxford: Oxford University.

[248] 39. Marsh, David \& Furlong, Paul. (2010). A Skin Not a Sweater: Ontology and Epistemology in Political Science. In: Marsh, David \& Stoker, Gerry (Eds.). Theory and Method in Political Science (pp. 184-211). New York: Palgrave Macmillan. https://doi. org/10.1007/978-0-230-36664-0_10

40. Monteiro, Nuno \& Ruby, Keven. (2009). IR and the False Promise of Philosophical Foundations. International Theory, 1 (1), pp. 15-48. https://doi. org/10.1017/S1752971909000050

41. Muller, Pierre. (2006). Las políticas públicas. Bogotá, D. C.: Universidad Externado de Colombia.

42. Orenstein, Mitchell. (2013). Pension Privatization: Evolution of a Paradigm. Governance, 26 (2), pp. 259-281. https://doi.org/10.1111/gove.12024

43. Pierson, Paul. (2000). Increasing Returns, Path Dependence, and the Study of Politics. The American Political Science Review, 94 (2), pp. 251-267. https://doi. org/10.2307/2586011

44. Roth, André-Noël. (2008). Perspectivas teóricas para el análisis de las políticas públicas: ¿de la razón científica al arte retórico? Estudios Políticos, 33, pp. 67-91.

45. Sabatier, Paul y Weible, Christopher. (2010). El marco de las coaliciones promotoras. Innovaciones y clarificaciones. En: Sabatier, Paul (ed.), Teorías del proceso de las políticas públicas (pp. 203-240). Buenos Aires: Proyecto de Modernización del Estado. 
46. Sankey, Howard. (1993). Kuhn's Changing Concept of Incommeusrability. British Journal of the Philosophy of Science, 44 (4), pp. 759-774. https://doi. org/10.1093/bjps/44.4.759

47. True, James; Jones, Bryan y Baumgartner, Frank. (2010). Teoría del equilibrio interrumpido. Explicación de la estabilidad y del cambio en la formulación de las políticas públicas. En: Sabatier, Paul (ed.). Teorías del proceso de las políticas públicas (pp. 167-202). Buenos Aires: Proyecto de Modernización del Estado.

48. Wilder, Matt. (2015). What Is a Policy Paradigm? Overcoming Epistemological Hurdles in Cross-Disciplinary Conceptual Adaptation. In: Hogan, John \& Howlett, Michael (Eds.). Policy Paradigms in Theory and Practice. Discourses, Ideas and Anomalies in Public Policy Dynamics (pp. 19-42). New York: Palgrave Macmillan. https://doi.org/10.1057/9781137434043_2

49. Wilder, Matt \& Howlett, Michäel. (2015). Paradgim Construction and the Politics of Policy Anomalies. In: Hogan, John \& Howlett, Michael (Eds.). Policy Paradigms in Theory and Practice. Discourses, Ideas and Anomalies in Public Policy Dynamics (pp. 101-116). New York: Palgrave Macmillan. https://doi. org/10.1057/9781137434043_6

50. Yanow, Dvora. (2014). Interpretive Analysis and Comparative Research. In: Engeli, Isabelle \& Rothmayr, Christine (Eds.). Comparative Policy Studies. Conceptual and Methodological Challenges (pp. 131-159). New York: Palgrave Macmillan. https://doi.org/10.1057/9781137314154_7

51. Yanow, Dvora \& Schwartz-Shea, Peregrine (Eds.). (2006). Interpretation and Method: Empirical Research Methods and Interpretive Turn. New York: M.E. Sharpe.

52. Zittoun, Philippe. (2009). Understanding Policy Change as a Discursive Problem. Journal of Comparative Policy Analysis, 11 (1), pp. 65-82. https://doi. org/10.1080/13876980802648235

53. Zittoun, Philippe. (2015). From Policy Paradigm to Policy Statement: A New Way to Grasp the Role of Knowledge in the Policymaking Process. In: Hogan, John \& Howlett, Michael (Eds.). Policy Paradigms in Theory and Practice. Discourses, Ideas and Anomalies in Public Policy Dynamics (pp. 117-140). New York: Palgrave Macmillan. https://doi.org/10.1057/9781137434043_7 